\title{
Tracking chloride and metal diffusion in proofed and unproofed concrete matrices using ablative laser technology (ICP-MS)
}

\author{
Avin Pillay ${ }^{1 *}$, Mirella Elkadi ${ }^{1 *}$, Fadi Feghali ${ }^{2}$, Sai Cheong Fok ${ }^{3}$, Ghada Bassioni ${ }^{4}$, Sasi Stephen ${ }^{1}$ \\ ${ }^{1}$ Department of Chemistry, The Petroleum Institute, Abu Dhabi, UAE; *Corresponding Author: apillay@pi.ac.ae \\ ${ }^{2}$ PO Box, 2431, Abu Dhabi, UAE; *Corresponding Author: melkadi@pi.ac.ae \\ ${ }^{3}$ Department of Mechanical Engineering, The Petroleum Institute, Abu Dhabi, UAE \\ ${ }^{4}$ Department of Chemical Engineering, The Petroleum Institute, Abu Dhabi, UAE. On leave from the Chemistry Department, Faculty \\ of Engineering, Ain Shams University, Cairo, Egypt
}

Received 8 May 2010; revised 17 June 2010; accepted 22 June 2010.

\section{ABSTRACT}

Depth profiling studies (laser ICP-MS) of ions $\left(\mathrm{Cl}^{-}, \mathrm{Na}^{+}, \mathrm{Mg}^{2+}\right)$ in concrete-based material can be used to provide useful information on the migration paths of these ionic species. In particular, deterioration of concrete through infiltration of chloride could lead to costly corrosion problems with serious impact on the environment. Many modeling studies on concrete matrices depend on the tortuosity of these transport paths. Our work showed that dispersion paths of ionic species in concrete are intermittent and sporadic, suggesting that applications of simplifying assumptions in treatment of such data could lead to appreciable perturbations in related mathematical models. This paper examines the capability of using a high resolution ICP-MS laser ablation technique to track $\mathrm{Cl}^{-} \mathrm{mi}-$ gration in concrete samples in the presence of other ions such as $\mathrm{Na}^{+}$and $\mathrm{Mg}^{2+}$. Cationic migration in such materials is underexplored and data in this particular area could contribute to modeling studies. Concrete bricks (with and without surface coatings) were specially prepared in cubic configurations and allowed to saturate in a ponding medium (sea water). The study subsequently examined the distribution of $\mathrm{Cl}^{-}, \mathrm{Na}^{+}$and $\mathrm{Mg}^{2+}$ with depth in protected (epoxy coated) and unprotected cored concrete slivers (5 mm diameter; $2 \mathrm{~mm}$ thick) using an $80 \mu \mathrm{m}$ diameter laser beam coupled to an ICP-MS instrument. The laser $(213 \mathrm{~nm})$ was programmed to ablate a total depth of $50 \mu \mathrm{m}$ at each point at $5-\mu \mathrm{m}$ intervals. The results in unprotected samples indicated that chloride intensity showed a general decline with depth, suggesting that mo- bility of the chloride is a function of its interaction with the concrete matrix. In some cases 'hotspots' were observed at certain points indicating that transport of the intruding ion was limited. No significant mobility was observed in coated samples. The depth-profiling results for $\mathrm{Na}^{+}$and $\mathrm{Mg}^{2+}$ were somewhat unexpected. Strong similarities in their spectra purported that the matrix was indifferent to charge and size of the ion. Our experimental data further showed that the matrix itself offers natural protection to the reinforced steel rebars by limiting chloride and metal diffusion at certain locations. Clearly, if the composition of these protective environments within the concrete could be simulated on a larger scale and introduced into the matrix it would offer scope for extended research in this area. Our work would be of definite interest to materials and environmental research; and mechanistic studies on aggregates.

Keywords: Concrete; $\mathrm{Cl}^{-}, \mathrm{Na}^{+} ; \mathrm{Mg}^{2+}$; Laser

Ablation; Depth-Profiling; ICP-MS

\section{INTRODUCTION}

In vitro tracking of chloride diffusion (by ICP-MS depth profiling) in the presence of multi-ionic species $\left(\mathrm{Na}^{+}\right.$, $\mathrm{Mg}^{2+}$ ) can provide information on two fronts: 1) the tortuosity of the migration pattern through the bulk material; and 2) the extent of diffusion in proofed concrete. It has been widely reported that concrete matrices incur serious environmental damage through invasive attacks of $\mathrm{Cl}^{-}$in the bulk material [1-5]. Concrete structures in contact with seawater or salt water are, therefore, expected to incur some form of environmental degradation. Oil and 
gas companies that have colossal concrete structures offshore tend to incur financial losses through salt-degradation of this nature. Salt sprays and splashes can also affect roadside concrete structures and buildings in the vicinity of the sea. The mechanism of corrosion due to $\mathrm{Cl}^{-}$invasion in concrete is still a subject of active study. It has been documented that when a concrete structure is exposed to seawater, chloride ions from this medium will gradually infiltrate the matrix, largely through the pores and fissures in the hydrated cement mixture [6]. Some migration studies have alleged that chloride ions linked to $\mathrm{MgCl}_{2}$ have different migratory properties than those associated with $\mathrm{NaCl}$ [7], and further work in this area would be useful. In the case of reinforced concrete, penetration of the chloride ions to the steel rebars, and subsequent accumulation to beyond a certain level, initiates corrosion in the steel, especially in the presence of moisture and oxygen at the steel-concrete interface.

Knowledge of the dispersion of $\mathrm{Cl}^{-}$and other species in a concrete matrix could contribute to mechanistic studies of ionic diffusion in such materials. Chloride mobility is particularly important and several factors could influence its dispersion pattern [8-11], such as: 1) the extent of transportation through the bulk material (by aqueous media); 2) salinity of the aqueous medium in contact with the concrete; 3 ) the level of interaction of the $\mathrm{Cl}^{-}$with chemicals and ingrained impurities in the concrete itself; 4) the porosity of the material; 5) temperature; 6) humidity; and 7) material homogeneity. A point to note about the last factor is that uneven mixing of the concrete could lead to considerable material inhomogeneity, which in turn could lead to aberrations in $\mathrm{Cl}^{-}$migration patterns.

We have developed an ultrasensitive technique for assessing the diffusion of $\mathrm{Cl}^{-}$(and cations such as $\mathrm{Na}^{+}$and $\mathrm{Mg}^{2+}$ ) in bulk concrete samples, and pinpointing areas in the matrix to study their spatial and depth dispersion. The technique uses laser ablation linked to a high performance ICP-MS instrument. Our work demonstrates that systematic depth profiling in concrete samples can provide clues to the tortuosity of the transport path, which could be useful in modeling studies. The aim of this paper, therefore, is to explore the potential of our method for rapidly tracking migration of $\mathrm{Cl}^{-}$and other ionic species in suitable bulk samples.

\section{MATERIALS AND METHODS}

\subsection{Sample Preparation/Sample Handling}

The cement samples used in this study were regular Portland cement material (Type 1), manufactured by the

${ }^{1}$ The Petroleum Institute, Abu Dhabi, UAE
Ras Al Khaimah cement plant in the United Arab Emirates (UAE) ${ }^{1}$. The concrete mix was designed using fine and coarse aggregates from the UAE. The maximum aggregate size was $10 \mathrm{~mm}$. The concrete mix preparation was based on the British Method Design (BRE 106). Two lots of concrete mixes were prepared to meet minimum target strengths of $20 \mathrm{~N} / \mathrm{mm}^{2}$ and $40 \mathrm{~N} / \mathrm{mm}^{2}$ (labeled Grade 20 \& Grade 40 respectively). The details of mix proportions are given in Table 1. Slump tests were conducted to ensure the practical workability of the concrete mixtures.

Bricks were cast from the two lots of concrete mixture. After casting, the bricks were cured under wet hessian blankets and polyethylene film. Compressive strength tests were performed on three concrete cubes $(150 \times 150$ $\times 150 \mathrm{~mm}$ ) from each lot of concrete mix after seven days to establish if the samples had attained the appropriate strengths. Table 2 shows the compressive strength results, which confirmed the quality of the cast concretes.

The cured bricks in each lot were further separated into two batches. Solvent free $100 \%$ epoxy resin (waterproof, chloride and carbonation resistant coating for protection of concrete) was applied to the bricks in one batch. The coating generally has excellent resistance to chemicals and UV exposure (i.e., suitable for the petrochemical industry) with an expected life span of about ten years. No coating treatment was applied to the bricks in the other batch. The two batches of coated and uncoated bricks in each lot were immersed in a pond of sea water. After several months, all the bricks were removed from the pond and samples were cored (discs of $5 \mathrm{~mm}$ diameter by $2 \mathrm{~mm}$ thick) from the bulk material using a standard coring tool [Makita TB131, Taiwan] (Figure 1). Laser experiments were conducted on coated and uncoated samples (bottom left and right of Figure 1, respectively).

\subsection{ICP-MS Laser Ablation Technology}

Samples were investigated with a Perkin Elmer SCIEX DRC-e ICP-MS (Connecticut, USA) fitted with a New Wave UP-213 laser ablation system. Laser ablation technology uses a micro-beam (from an Nd: YAG solid state laser) to ablate samples in a special sample chamber. The fine ablated material is transported by a carrier gas (Ar) to a hot plasma where it is atomized and converted to ions (characteristic of the elements of the sample), which are subsequently carried to a mass spectrometer for detection (Figure 2).

The extremely high temperature of the plasma (about $10,000 \mathrm{~K})$ separates the sample into individual atoms. The plasma subsequently ionizes these atoms $\left(\mathrm{M} \rightarrow \mathrm{M}^{+}\right.$ $\left.+\mathrm{e}^{-}\right)$so that they can be detected by the mass spec- 
Table 1. Concrete mix proportions.

\begin{tabular}{|c|c|c|c|c|c|}
\hline \multirow{2}{*}{$\begin{array}{l}\text { Concrete Targeted } \\
\text { Strength }\left(\mathbf{N} / \mathbf{m m}^{2}\right) \\
\end{array}$} & \multicolumn{5}{|c|}{ Mix Proportion } \\
\hline & Cement kg/m $\mathrm{m}^{3}$ & Water Cement Ratio & Density $\mathrm{kg} / \mathrm{m}^{3}$ & Aggregate Cement Ratio & $\%$ Fine aggregate \\
\hline 20 (Grade 20) & 320 & 0.54 & 2409 & $5.98: 1$ & 53.0 \\
\hline 40 (Grade 40) & 400 & 0.41 & 2440 & $4.68: 1$ & 52.0 \\
\hline
\end{tabular}

Table 2. Compressive strength of concrete mixes.

\begin{tabular}{|c|c|c|c|c|}
\hline \multirow{2}{*}{$\begin{array}{l}\text { Concrete Targeted } \\
\text { Strength } \mathbf{N} / \mathbf{m m}^{2}\end{array}$} & \multicolumn{3}{|c|}{ Compressive strength after 7 days $\left(\mathrm{N} / \mathrm{mm}^{2}\right)$} & \multirow{2}{*}{$\begin{array}{c}\text { Average Measured Compressive } \\
\text { Strength } \mathrm{N} / \mathrm{mm}^{2}\end{array}$} \\
\hline & Cube 1 & Cube 2 & Cube 3 & \\
\hline 20 (Grade 20) & 37 & 38 & 37.5 & 37.5 \\
\hline 40 (Grade 40) & 48 & 50 & 53 & 50 \\
\hline
\end{tabular}
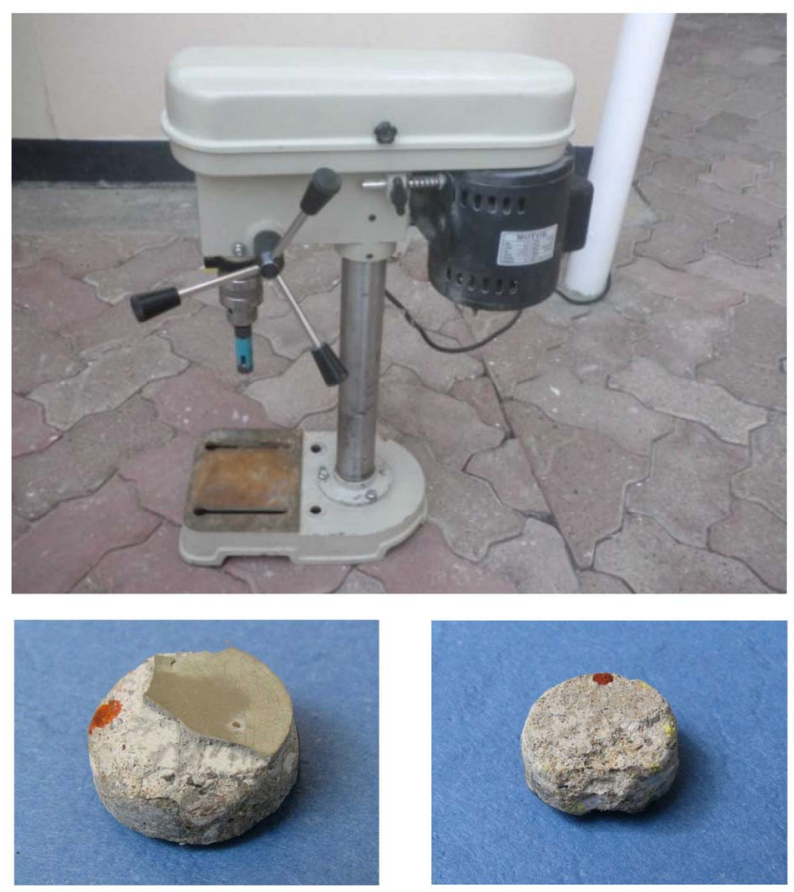

Figure 1. The coring instrument (top); and typical samples of proofed and unproofed cored discs (bottom left and right, respectively).

trometer. The technique is highly sensitive and can attain a limit of detection of $10^{-6} \mathrm{mg} / \mathrm{kg}$ (parts per trillion) for most elements. The concrete cored discs were placed into a special sample holder with dimensions $5 \mathrm{~cm} \times 5 \mathrm{~cm}$. No serious pre-treatment was necessary prior to irradiation. Samples were subjected to 213-nm laser irradiation at different points on the sample (16-point grid-Figure 2). The level of the beam energy was $30 \%$, with a fluence of approximately $3 \mathrm{~J} / \mathrm{cm}^{2}$ and beam diameter of 50 $\mu \mathrm{m}$. The laser was programmed to ablate a depth of 5 $\mu \mathrm{m}$ at each point and repeatedly scanned the surface; recording measurements after each ablation to a total depth of $50 \mu \mathrm{m}$.

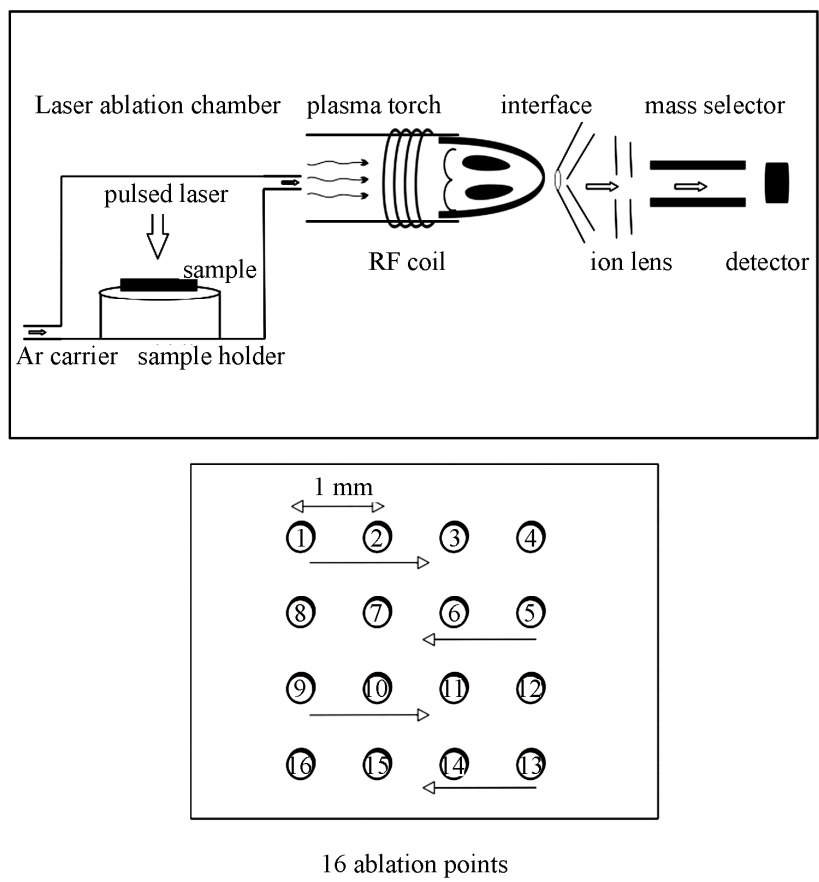

Figure 2. The ICP-MS instrument (top); 16-point grid showing the arrangement of points on a sample irradiated by the laser (bottom).

\subsection{Instrumental Performance}

Characteristic intensities originating from the elements of interest were measured; and valid considerations were given to potential interferences and matrix effects. Prior to each run, the performance of the instrument was validated $[12,13]$. The study was largely semi-quantitative in the absence of standardization, and for purposes of comparison, all measurements were conducted under identical experimental conditions. Appropriate spectra were produced to observe variations in characteristic elemental profiles spatially and with penetration depth. A point to note is that the ponding medium produced $\mathrm{Cl}^{-}$, 
$\mathrm{Na}^{+}$and $\mathrm{Mg}^{2+}$ ions which are in elemental form in the solid matrix. Validating the analytical performance of the laser technique was conducted on an available certified NIST standard (National Institute of Standards and Technology, Maryland, USA; Certificate 613, glass bead). To ascertain that the instrument was optimally functional we examined its performance for different isotopes by taking replicate measurements $(n=3)$ for equivalent counting times at random points on the standard. Relative standard deviations of less than $5 \%$ were attained in general (Table 3) indicating that the operational performance of the instrument was acceptable. It is necessary to underscore that in the absence of matching-matrix standards our method was based on evaluating relative intensities (counts/sec) for purposes of comparison.

\section{RESULTS AND DISCUSSION}

\subsection{Chloride Diffusion in Unprotected Concrete Matrices-Depth Profiling}

Laser ablation technology is capable of depth and surface analysis, displays the elemental (ionic) intensities and produces an elemental profile [14]. Ablative technology is particularly useful for the measurement of ultra-trace intensities of metals in solids. Chloride detection is more energetically demanding due to the higher ionization potential of chlorine; therefore, the technique is limited to determination of minor concentrations of this element. Interferences from the matrix are generally common in ICP-MS, but with the availability of sophisticated software and collision reaction cells they are becoming easy to detect and handle.

Depth profiling is a special process to investigate elemental distribution beneath the surface. Very few contemporary instrumental methods have the capability to study metal intensity with depth [12]. X-ray methods are useful, but lack the ability to control depth penetration. Nuclear particle irradiation is equally useful, but such techniques require nuclear accelerators, and tend to be limited to only a few microns below the surface. The competence, therefore, of the laser approach to delve to discreet depths below the surface of a sample is attractive for homogeneity studies in bulk materials. A typical spectrum depicting the profile of chloride with depth appears in Figure 3(a). The general trend portrays oscillating intensities suggesting that migration of chloride through the bulk material leads to interaction with the matrix. Similar trends appeared in both sample grades. Such interaction ultimately results in undesirable metamorphosis of the sample [15] causing material damage [16]. Clearly, the path of migration through the concrete would depend on its compositional make-up. The spec- trum in Figure 3(a) shows that at certain depths the chloride intensity changes sharply. This could be due to minor 'blockages' that limit permeability and create a diversion in the migration path itself. These blockages could be made up of conglomerates of gravel/sand/cement in certain proportions and knowledge of such constitutions would be useful to limit the infiltration of chloride through the bulk material.

A good example of an obstructed path is shown in Figure 3(b), which represents a spectrum delineating an abrupt end to the diffusion of chloride after a depth of about $25 \mu \mathrm{m}$. Evidently, some obstruction in the migration path at a depth of $25 \mu \mathrm{m}$ prevented the chloride from travelling any further. The data in Figure 3(b) suggests that either permeability is limited or chloride migration stops. The mechanistic details are difficult to devise and it is not clear at this stage exactly how permeability and chloride migration are linked. The spectrum in Figure 3(c) is interesting for the simple reason that it shows dispersion of chloride up to $22 \mu \mathrm{m}$ and then a blocked path between 22-32 $\mu \mathrm{m}$ followed by continued migration beyond a depth of $32 \mu \mathrm{m}$. This continued migration could be attributed to either another stream of chloride ions that overlapped with the track of the laser beam; or the same stream that went via another route and again aligned itself with the main stream. This spectrum in particular delineates the tortuosity of the transport path, which if understated could lead to deviations in related modeling studies.

\subsection{Spatial Variation of Chloride}

Iterative surface scans provided useful information on the distribution of chloride on the surfaces of the concrete samples. Figure 4 presents a bar-graph showing the typical variation of chloride intensity at different ablation points on the surface of a selected sample. For convenience, points on the plot represent maximum to minimum intensities to exemplify the dramatic fluctuation of chloride intensity.

The reason for such sharp variations on the surface is not clear and could possibly be attributed to the inhomogeneous nature of the matrix itself. The pronounced difference in intensity by as much as a factor of about 20 would indicate that there are sites on the surface that are

Table 3. Measurements (counts/sec) of reproducibility in a NIST 613 standard.

\begin{tabular}{ccccccc}
\hline Measurement & ${ }^{59} \mathrm{Co}$ & ${ }^{85} \mathrm{Rb}$ & ${ }^{51} \mathrm{~V}$ & ${ }^{138} \mathrm{Ba}$ & ${ }^{140} \mathrm{Ce}$ & ${ }^{238} \mathrm{U}$ \\
\hline 1 & 567 & 1700 & 2900 & 7069 & 1333 & 1167 \\
2 & 567 & 1767 & 2900 & 7103 & 1367 & 1200 \\
3 & 633 & 1733 & 3101 & 7069 & 1367 & 1267 \\
Mean \pm & $589 \pm$ & $1733 \pm$ & $2967 \pm$ & $7080 \pm$ & $1356 \pm$ & $1211 \pm$ \\
RSD & $5.2 \%$ & $1.6 \%$ & $3.2 \%$ & $0.23 \%$ & $1.2 \%$ & $3.4 \%$ \\
\hline
\end{tabular}



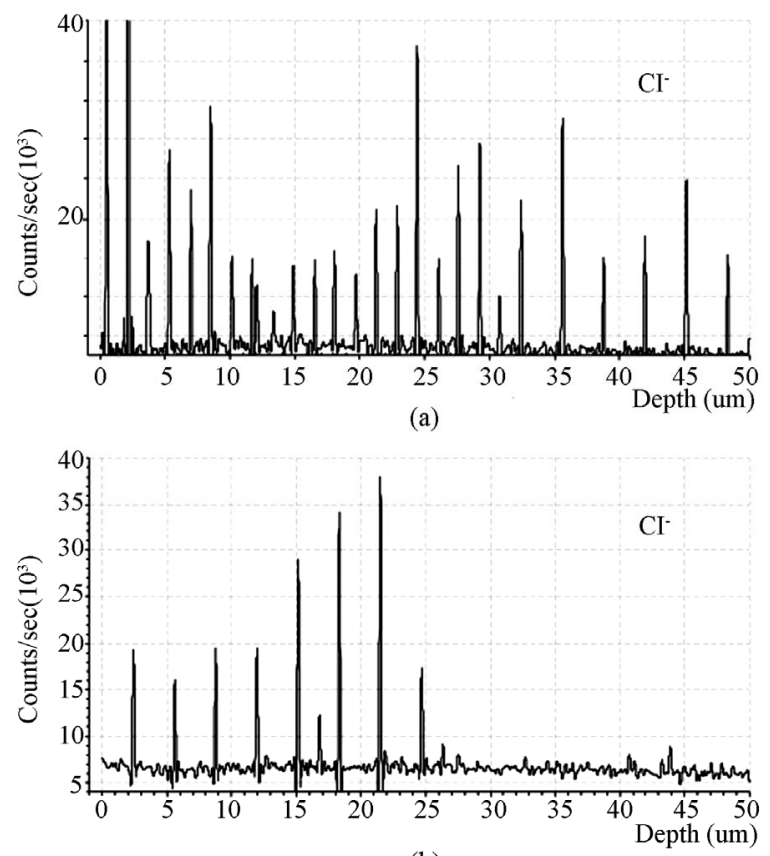

(b)

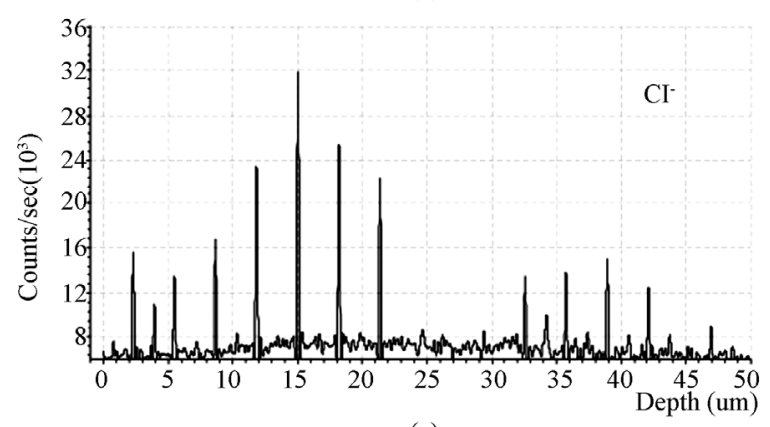

(c)

Figure 3. Depth-profiling spectra of chloride: (a) an example of an unbroken path (Grade 20); (b) an example of a blocked path (Grade 20); and (c) an example of a path that is blocked between 22-32 $\mu \mathrm{m}$ and continued chloride migration thereafter (Grade 40).

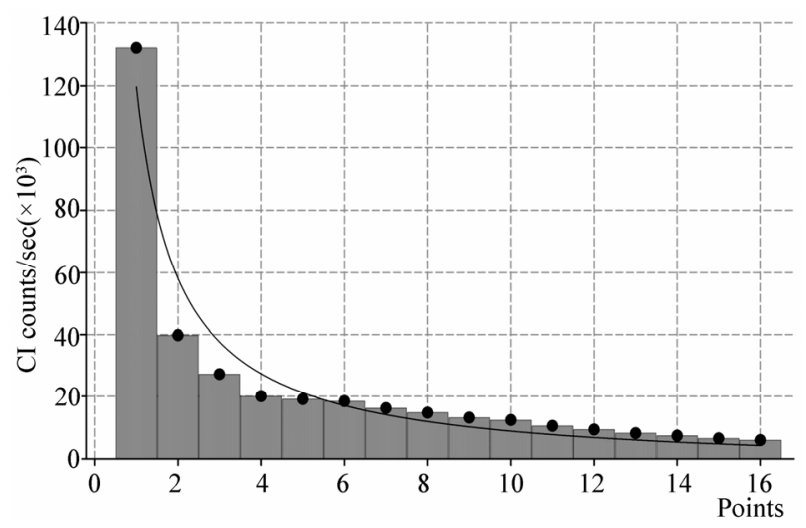

Figure 4. Typical spatial distribution of chloride on sample surface. prone to accumulation of chloride-whereas at other points chloride can easily infiltrate the sample (through the medium of the ponding solvent). It would be of considerable interest to identify the locations on the surface matrix that tend to inhibit chloride diffusion. From the accumulated data it would seem that the matrix itself offers natural protection to the reinforced concrete by limiting chloride diffusion at certain spots. A knowledge of the composition of those inhibiting sites would be useful and if simulated could be converted into a chemical additive or inhibitor to serve as a protective layer.

\subsection{Hotspots/Degradation Problems}

The appearance of sporadic abnormally tall peaks in a spectrum demonstrates the presence of 'hotspots', (Figure 5). 'Hotspots' are sites in the interior of the sample where chloride 'agglomerates'. Once again, such accumulation of chloride ions could be due to several factors. Sudden blockages in the diffusion path; 'bottlenecks;' pores and pockets where the ponding solvent decelerates in its progressive dispersion could collectively be responsible for the 'hotspot' phenomenon. The mechanism linked to corrosion of reinforced steel is difficult to define. However, reports in the literature suggest that chloride plays a key role in the degradation process [6]. Chloride tends to weaken and destroy the thin coatings of iron oxides on the rebars. The steel is subsequently exposed to oxygen and moisture and begins to corrode. It is known that a minimum level of chloride is necessary to initiate corrosion $[6,17]$. Our data indicate that 'hotspots' are convenient internal sites in the matrix where chloride ions have the opportunity to accumulate and contribute to internal degradation. Such interactions combined with increased stress in the material lead to delamination in the matrix, which subsequently results in staining of the concrete. Typical staining resulting from these factors appears in one of our samples shown in Figure 6.

\subsection{Proofed Concrete}

Most protective layers in concrete tend to shield it from internal degradation. Effective proofing on concrete should be impervious to infiltration of chloride ions. Figure 7(a) represents a depth-profile spectrum of the proofing itself. Clearly, no chloride or other metals are present. The proofing itself works efficiently provided no cracks or fissures exist in the coating. A point to bear in mind is that minor fissures and cracks permit permeation of the ponding solvent and access of chloride into the sample. A meticulous scan of the coating was made with the laser and Figure 7(b) represents an imperfection in the epoxy where the ponding medium infiltrated the coating and traces of chloride were seen. In general the 


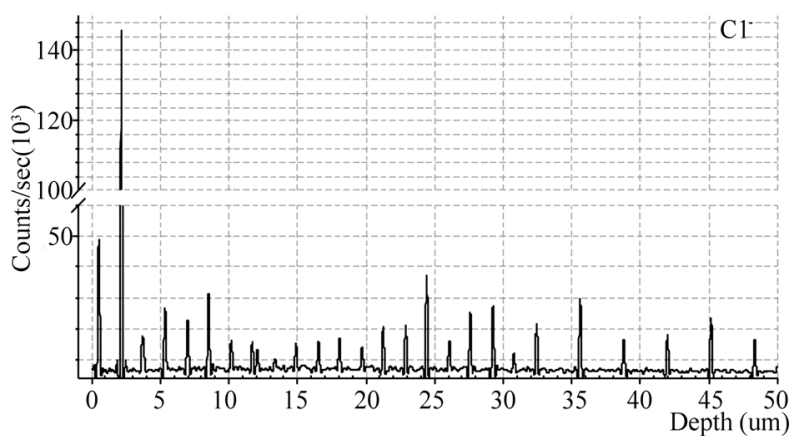

Figure 5. Example of 'hotspot' in depth-profiling spectrum of chloride (Grade 20).

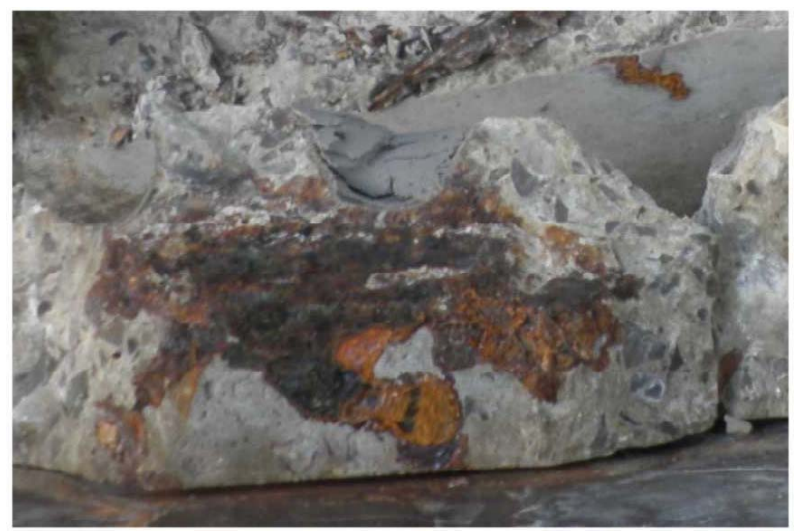

Figure 6. Staining and corrosion damage due to invasive attack of chloride.
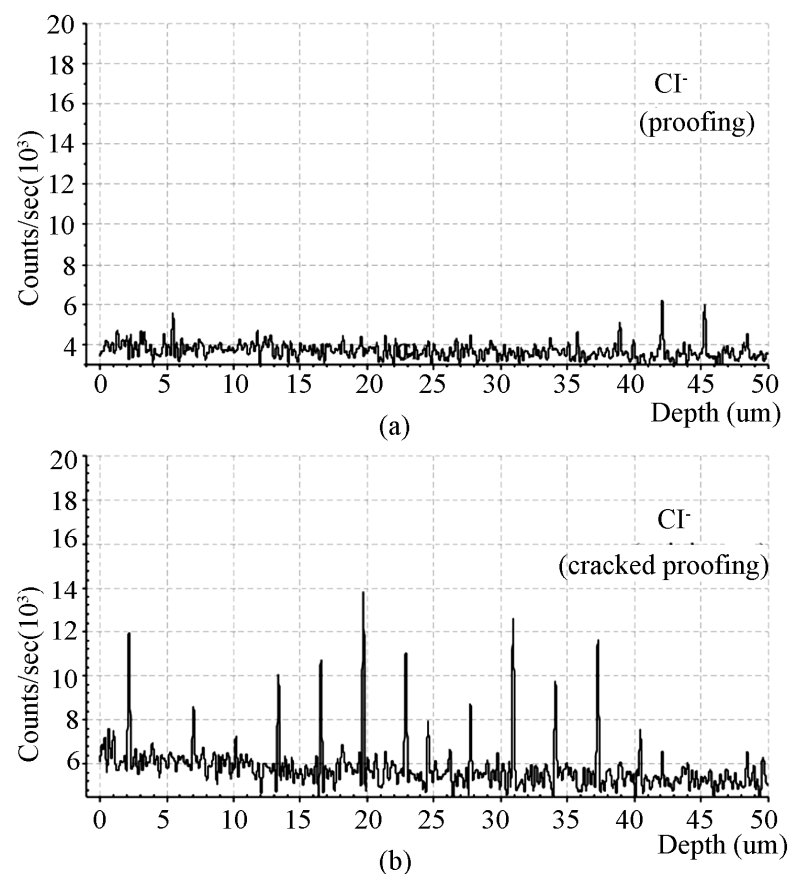

Figure 7. (a) Depth-profiling spectrum of epoxy proofing (Grade 20); (b) depth-profiling spectrum of cracked epoxy proofing showing chloride infiltration (Grade 40). absence of chloride would proclaim the efficiency of the proofing. However, as with all epoxies exposure to a combination of solar radiation and sea water tends to cause the surface to 'chalk' and degrade a few microns each year. Hairline cracks are formed in this way through which the immersion solvent can gain free passage.

\section{5. $\mathrm{Na}^{+}$and $\mathrm{Mg}^{2+}$ Diffusion}

A report by Mussato et al. [7] suggests that the migratory properties of chloride ions originating from $\mathrm{MgCl}_{2}$ differ from those linked to $\mathrm{NaCl}$. Whether or not this theory has been verified is not clear, but our work tends to lend credence to the view that differences in such transport properties could exist. Figures 8(a) and 8(b) represent typical depth profiling spectra of $\mathrm{Na}^{+}$and $\mathrm{Mg}^{2+}$ respectively in the unproofed sample. Similar trends appeared in both sample grades. Control samples showed no serious levels of ionic species.

Both spectra seem to mimic each other. This was an unexpected development and purported that the matrix 'adsorbs' both cations concurrently irrespective of size and charge of the ion. It could also suggest that there are points or 'bottlenecks' within the matrix where physical conditions promote supersaturation of the salts of these metals thus leading to their agglomeration and the spectral similarities observed in Figure 8. However, of significance is that the level of sodium chloride is about five times higher than magnesium chloride in sea water and a comparison of the heights of the peaks in Figures

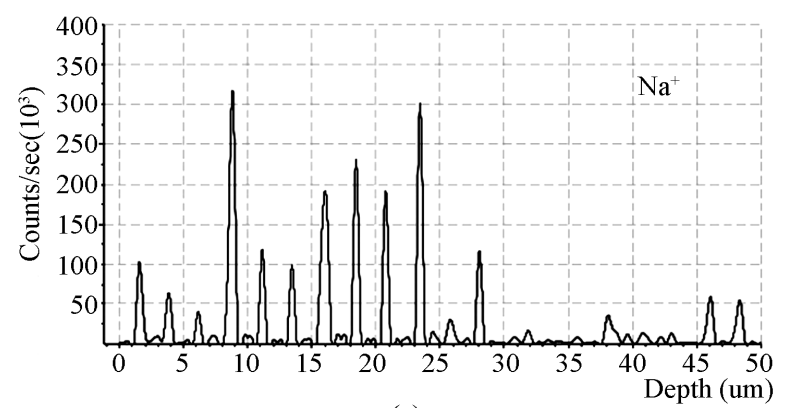

(a)

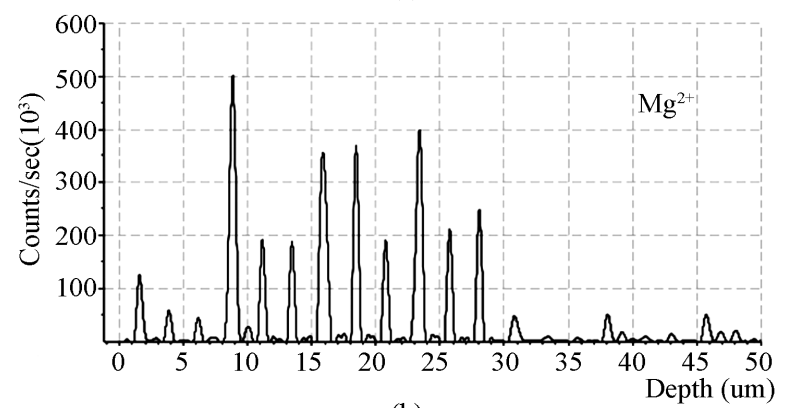

Figure 8. Typical depth-profiling spectra of a Grade 20 sample: (a) sodium; and (b) magnesium. 
8(a) and 8(b) indicate that the intensities of $\mathrm{Mg}^{2+}$ and $\mathrm{Na}^{+}$do not vary appreciably, indirectly suggesting that transport differences linked to chloride could exist and should be more closely investigated. Both spectra also delineate areas of high and diminished intensity. The areas of low intensity indicate that the region in the interior of the sample is not conducive to 'adsorption' of the metal cations, and could be attributed to meager interaction with the matrix. As in the case of chloride, the areas of high intensity suggest that the metals linger due to some interaction with the matrix. Figures 9(a) and 9(b) portray typical depth-profiling spectra of a proofed sample. Again the spectrum of $\mathrm{Na}^{+}$mirrors that of $\mathrm{Mg}^{2+}$. Both spectra (Figure 9) reveal peaks up to a depth of $10 \mu \mathrm{m}$ suggesting that the ponding medium penetrated the proofing and left traces of these metals on the surface. This leads us to believe that such penetration could have taken place through a hairline crack or fissure as we observed in Figure 7(b), and that the unbroken proofing itself was impervious to the seawater.

\section{CONCLUSIONS}

Our experimental data showed that the matrix itself offers natural protection to the reinforced steel rebars by limiting chloride and metal diffusion at certain locations. Clearly, if the composition of these protective environments within the concrete could be simulated on a larger scale and introduced into the matrix (in the form of additives or inhibitors) it would offer scope for extended

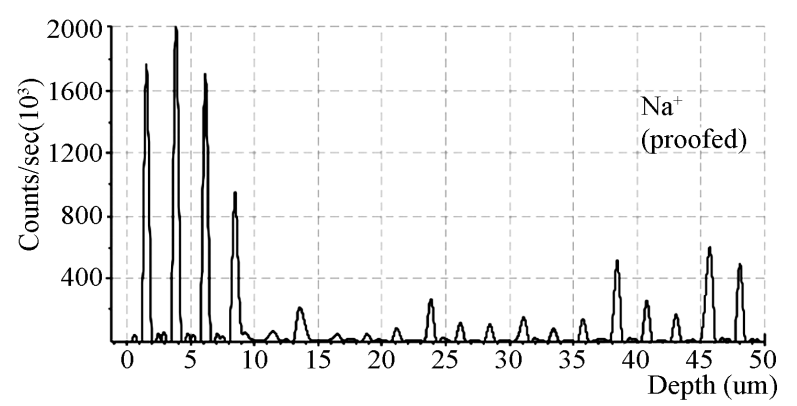

(a)

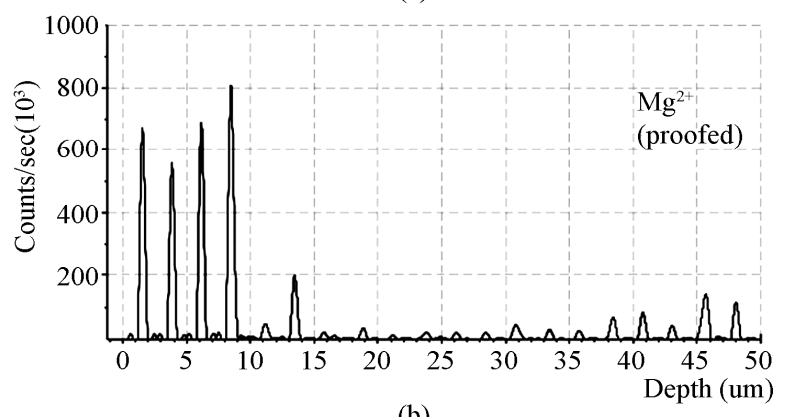

(b)

Figure 9. Spectra of $\mathrm{Na}^{+}$and $\mathrm{Mg}^{2+}$ in the proofed sample (Grade 40) showing infiltration up to about $10 \mu \mathrm{m}$. research in this area. Unquestionably, adding 'chemical inhibitors' to the concrete mixtures could be a useful way to control the rate of corrosion (provided that they complement the natural function of the matrix in shielding the steel rebars). Exploring the possibility of differences in migratory properties of chloride ions associated with $\mathrm{MgCl}_{2}$ and $\mathrm{NaCl}$ could also be the subject of future study.

\section{AKNOWLEDGEMENTS}

The authors thank the petroleum institute for financial assistance.

\section{REFERENCES}

[1] McPolin, D., Basheer, P.A.M., Long, A.E., Grattan, K.T.V. and Sun, T. (2005) Obtaining progressive chloride profiles in cementitious materials. Construction and Building Materials, 19(9), 666-673.

[2] Meck, E. and Sirivivatnanon, V. (2003) Field indicator of chloride penetration depth. Concrete and Concrete Research, 33(8), 1113-1117.

[3] Tang, L. and Nilsson, L.O. (1992) Rapid determination of the chloride diffusivity in concrete by applying an electrical field. ACI Materials Journal, 89(1), 49-53.

[4] Tang, L. (1996) Electrically accelerated methods for determining chloride diffusivity in concrete-current development. Magazine of Concrete Research, 48(176), 173-179.

[5] Yang, C.C. (2004) The relationship between migration coefficient of chloride ions for concrete and charge passed in steady state using the accelerated chloride migration test. ACI Materials Journal, 101(2), 124-130.

[6] Rosenberg, A.M. and Gaidis, J.M. (2001) Avoiding corrosion damage in reinforced concrete. Concrete International, 23(11), 80-83.

[7] Mussato, T., Gepraegs, O.K. and Farnden, G. (2004) Relative effects of sodium chloride and magnesium chloride on reinforced concrete: State of the art. Journal of the Transportation Research Board, 1866, 59-66.

[8] Climent, M.A., de Vera, G., Lőpez, J.F., Viqueira, E. and Andrade, C. (2002) A test method for measuring chloride diffusion coefficients through non-saturated concrete, Part I: The instantaneous plane source diffusion case. Cement and Concrete Research, 32(7), 1113-1123.

[9] Yamada, Y., Oshiro, T. and Masuda, Y. (1999) Study on chloride penetration into concrete. Summaries of Technical Papers of Annual Meeting, AIJ, Japan, September, 1999, 961-962.

[10] Ulrich, S. (1988) Concrete at high temperatures. Fire Safety Journal, 13(1), 155-168.

[11] Friedmann, H., Amiri, O., Aït-Mokhtar, A. and Dumargue, P. (2009) A direct method for determining chloride diffusion coefficient by using migration test. Cement and Concrete Research, 34(11), 1967-1973.

[12] Robinson, J.W., Skelly-Frame, E.M. and Frame, G.M. (2005) Undergraduate instrumental analysis. 6th Edition, Marcel Dekker, New York.

[13] Jarvis, K.E., Gray, A.L. and Houk, R.S. (1992) Handbook 
of ICP-MS. Blackie Publishers, Glasgow.

[14] Gastel, M., Becker, J.S., Kuppers, G. and Dietze, H.J. (1997) Determination of long-lived radionuclides in concrete matrix by laser ablation inductively coupled plasma mass spectrometry. Spectrochimica Acta Part B: Atomic Spectroscopy, 52(14), 2051-2059.

[15] Bassioni, G. and Plank, J. (2006) Untersuchungen zur kompetitiven adsorption anorganischer anionen am modellsystem kalksteinmehl. GDCh Monographie, 36, 225-232.
[16] Plank, J., Bassioni, G., Dai, Z., Keller, H., Sachsenhauser, B. and Zouaoui, N. (2006) Neues zur Wechselwirkung zwischen Zementen und Polycarboxylat-Fließmitteln. Ibausil-Tagungsband, 16, 579-598.

[17] Bassioni, G. (2009) Studies on halide-induced corrosion on different types of steel. International Reviews in Chemical Engineering, 1(6), 547-551. 